\title{
(a) OPEN ACCESS
}

\section{Mobile health application platform 'Corrie' personalises and empowers the heart attack recovery patient experience in the hospital and at home for an underserved heart attack survivor}

\author{
George Hung, ${ }^{1,2}$ William E Yang, ${ }^{1,2}$ Francoise A Marvel, ${ }^{1,2}$ Seth S Martin ${ }^{1,2}$
}

${ }^{1}$ Department of Medicine, Johns Hopkins University School of Medicine, Baltimore, Maryland, USA

${ }^{2}$ Ciccarone Center for the Prevention of Cardiovascular Disease, Division of Cardiology, Department of Medicine, Johns Hopkins Hospital, Baltimore, Maryland, USA

\section{Correspondence to}

Dr George Hung;

ghung3@jhmi.edu

Accepted 14 January 2020

Check for updates

(c) BMJ Publishing Group Limited 2020. Re-use permitted under CC BY-NC. No commercial re-use. See rights and permissions. Published by BMJ.

To cite: Hung G, Yang WE, Marvel FA, et al. BMJ Case Rep 2020;13:e231801. doi:10.1136/bcr-2019231801

\section{SUMMARY}

Cardiovascular disease (CVD) is the leading cause of morbidity and mortality worldwide with an estimated 17.5 million deaths annually, according to the World Health Organization (WHO). CVD prevention efforts have the potential to prevent the majority of these deaths by supporting guideline-directed medical therapy (GDMT) and lifestyle modification. Mobile health (mHealth) has the potential to address this gap, but has limited evaluation in clinical studies to date. We present the case of a middle-aged patient of low socioeconomic status, with multiple comorbidities, and no prior smartphone experience, who suffered an acute myocardial infarction (MI) and was given the Corrie intervention while hospitalised. The patient demonstrated improvement in lifestyle modification, adherence to GDMT and post-MI recovery through 2.4 years follow-up. This case supports (1) the potential of mHealth interventions to enhance patient experience and outcomes, (2) intuitive design for adoption and improvement in end user experience and (3) the capability of mHealth to reach and empower underserved patients

\section{BACKGROUND}

At present, cardiovascular disease prevention efforts after a myocardial infarction (MI) are performed via standard paper-based discharge instructions at the time of hospital discharge. This critical event for post-MI recovery involves multiple hand-offs and inconsistent information delivery requiring high health literacy, much of which may be delivered in the final minutes of hospitalisation. Recent studies investigating hospital discharge found $81 \%$ of patients had an unclear understanding of their medications, ${ }^{1} 48 \%$ had uncertain follow-up ${ }^{1}$ and $40 \%$ had difficulty describing the reason for hospitalisation. $^{2}$ To address these gaps, mobile health (mHealth) interventions have emerged to help engage patients in managing their health. Although mHealth presents opportunities to re-engineer the hospital-to-home transition, study participants are generally younger, healthier and of higher socioeconomic status, ${ }^{3}$ raising questions around its benefit to underserved populations at greater risk of poor health outcomes. We present a vulnerable patient with multiple cardiovascular risk factors and social barriers to healthcare, who derived great benefit

(see the Patient's perspective section) from an mHealth intervention for MI survivors.

\section{CASE PRESENTATION}

A 55-year-old woman with undiagnosed familial hypercholesterolaemia, pre-diabetes, tobacco use, physical inactivity, diet consisting of fried and processed foods, and morbid obesity was admitted with chest pain. She was diagnosed with an inferior ST-elevation MI and two drug-eluting stents were placed in her right coronary artery. She had previously been incarcerated, earned US $\$ 31000$ annually working at a retail store, and had been uninsured for several years. She had never received preventive care to reduce her cardiac risk factors.

A

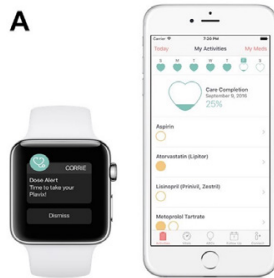

C
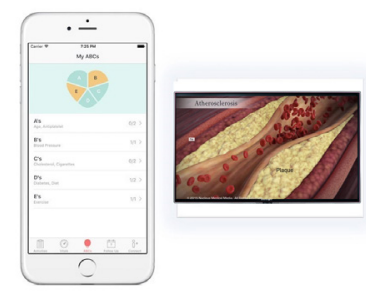

Figure 1 Corrie iPhone and Apple Watch app for patients with acute myocardial infarction. (A) Medication adherence tracking and reminders; $(B)$ vital signs monitoring, integrated with Apple Health. Realtime monitoring of heart rate and step count with the Apple Watch via Apple Health; (C) educational modules including videos, animations via Nucleus Media and articles at the 6 th grade reading level. Completing modules is incentivized by 'Heart of Gold' reward; (D) Healthcare provider directories and appointment reminders which integrate with Apple Calendar. This figure was created by the coauthors with key technical assistance from Corrie engineering and Lead iOS Developer Ryan Demo. Note: Corrie is also available on all newer iPhone and Apple Watch models. 
Patient's perspective

The beginning of her heart attack story: the shock and overwhelming experience of going through a myocardial infarction:

When I had the heart attack, [I was] scared, they're telling my friend you've got to go to the waiting room, because she needs to get to the OR. [After the procedure] When I was in my hospital room... maybe a day went by [and] no matter what was going on and who was talking to me, I wasn't comprehending half of what anybody was saying, I was still blown away from the heart attack. I was thinking of different stuff, as I thought of different stuff, I really wasn't getting anything accomplished thinking about it because my mind was everywhere and nowhere all at the same damn time. You don't feel safe after a heart attack. I was afraid to go to sleep. My days and nights were jacked up. I went to a shrink [later on].

\section{Her introduction to Corrie:}

So then, in walks [my] doctor. I had been trying to think, I have always lived my life, that I have to have a purpose to do something, if I have a purpose in my life, I do better. And you started talking about the program and the benefits of [Corrie]. What I said was, I didn't know, but I would try [the Corrie program]. And so that night, when my roommate came to visit me, I told her what you said, [Corrie] would keep track of everything from steps to heart beats. They should give one to everybody! I did every one [of the Corrie education videos] in the first night. I watched them all before I left the hospital.

Her learning new technology with the help of caregivers:

I'm naïve, I didn't know nothing about technology, this technology, I went to school in the 80 s, that computer tech don't mean a darn thing anymore, DOS is dead, all that stuff is done. So for me, the only technology I had was my flip phone. He taught me different ways to youtube and stuff. My partner's son. He came over because he's more tech. He thought it was cool, because mine was better than the one he paid 900 dollars for. It gets them involved, because he thought it was the coolest thing.

Her post-discharge experience with Corrie: quitting smoking, exercising, losing weight:

Now I have a purpose of what I'm going to do when I go home. I quit smoking, which was a huge huge obstacle. I went and got the vapor cigarette, only got the 12 point milligram nicotine. I couldn't stop cold turkey, I knew I wouldn't make it. I was doing everything that that watch and the telephone and you told me to do. We had some issues, because nobody would let me go to the cardio-workout that you guys wanted me to do because of insurance purposes. So I came every night and walked up and down these steps and did my own cardio work-out. Every time I got to the top it was reason to cheer. I went from 164 to $124 \mathrm{lbs}$ when I was working the program.

I wanted to get used to it in the house before I went back to work. I was doing it up and down the street around the block, and then 2 weeks later when cardio people [rehab insurance representative] said no, I was over here running the steps. The tools helped, I learned how to do the tools.

Her post-discharge experience with Corrie: diet changes:

We eat a lot of chicken now, I bake everything, there is no fried food, 1 day a week is a fried food day, because it used to be 5 days fried food, 2 days baked. So food has changed, because of the videos, and I got cookbooks from work, e.g. 'eating healthy living longer'. In the videos it says you can get this book, or that

\section{Patient's perspective Continued}

book. I'll get a couple of marked down cookbooks at work. I took fresh salmon, on the side you mix up mustard, balsamic vinegar, salt, pepper, it tastes so good, oh my lord, makes you wanna slap your mama. We only get one day to cheat, only because we have the niece and the [young child]. We have lots of fish dishes. I took fresh green beans with just pepper and oregano, and fake sea salt. And that's all you had, and it was good. So yeah, we eat a lot better. But it's not the app, it's the program, Corrie is the heart of the whole thing working.

Her difficult times amidst tragedy: return to smoking:

And then in comes the grandson passing away of my partner... he's killed in a tractor trailer accident. It's his day to train somebody, and he told the boss he's not ready and the boss said take him anyway. And he kills him right there on the road. And I go with the partner to Shock Trauma because they don't tell him he's dead. Now mind you I have my watch on, and it's beating pretty fast. So there's a lot of stuff going, all of her family which I'm very close to, everybody's crying, the daughter's laying on the ground, she can't take it that's her son, her only son, ... with a wife and 1 child, and she's pregnant with her next child, due any day, and she's a mess. So the waiting room is all the family. So she starts smoking, I light up a cigarette, and all hell breaks loose. Because at a certain point, there was way too much stress for me because she was gonna lose it. My concern at that time, when I was in the coma for 12 days, she sat every day with me, so I wanted to be there for her, and whatever she needed she was gonna get it, but I needed the cigarette, because nobody was there for me. And I was like, I'm going to be better, but this is what I need to do. So I faltered there for a few months.

Her having to part ways from Corrie:

'When I got the call to return the equipment [after the tragedy], it felt like I was losing a kid. Return [timing] should come from the patient when they are ready to move on. Had I had it, it wouldn't have taken me as long to get back into it. It should be up to the patient when they feel like they're there. It works as a tool for you to continue to want to get better, and to get better.'

Her reinvigoration with the return of Corrie:

When [my] doctor walked in and said here's your watch and phone, I was like, this on my way home! Now I'm down to 4 cigarettes per day. I'm getting back to getting ready to stop again. [My] doctor brought me the watch and the phone back $\ldots$ and that gave me purpose number 2 to go back to what I was doing. I weighed 166 lbs when he gave me the phone, I'm down to $136 \mathrm{lbs}$. I'm working the program, even when the watch doesn't work for me.

Her comparison of normal hospital discharge to discharge with Corrie:

The difference was you had something in your hand. I read everything on the Apple phone before doing anything, because you kept hitting different stuff, you could read and read. It explained different things, about the phone, about what Corrie was, about what the functions were of different things. I've always been a hands on kind of person, not a reader so I would rather have a tool, than to read about a tool. If I was reading a bunch of paperwork about I had to do this exercise, this exercise, and this exercise, those papers would stay sitting by the couch, and I probably wouldn't have done anything. You know what I'm saying. It wouldn't have motivated me to get up. But when I saw that the watch watched me going to the bathroom and kitchen, 
Patient's perspective Continued

was tracking steps, I wanted to see how far the steps would go. I was like, maybe I can do this. So, It just got more exciting and more exciting. It just built up, it was crazy.

I felt it gave a purpose to get off the couch. If there would've been a stack of papers I probably would've stayed on the couch, because they gave me a stack of papers, I never look at them. I look at the watch.

As soon as you start seeing it work, that's when you're going to want to do better and better and better. I know your brain is everywhere else, but this will keep your brain from worrying about what you're not doing. Because right now, you had a heart attack, and your life is never going to be the same, I'm not going to lie. You're not gonna walk the same walk, you're not even going to talk the same way. But you will get better, if you just try.

\section{Her hospital discharge experience in general:}

It was only 2 days, then they were getting ready to release me, which I thought was kinda fast, I thought it was too fast, I almost didn't want to leave. But that's what the insurance company said. She can go home and lay on a couch. At that time [after my MI], my partner was afraid of everything I did. She wouldn't let me clean the house or sweep the floor.

\section{Her perspective on what could be better:}

But the obstacles are what you gotta look at. 9 times out of 10 , that patient is going to able to do what you ask them to do. But you're going to run into that $10^{\text {th }}$ patient that because of her heart attack, she loses her insurance [i.e. our patient here], and it goes from 57 dollars a [bill] to 287 dollars a [bill]. The state says she makes too much. [The hospital representative] says they can't put you on a finance plan. I can't get into the office to see nobody. Those are the things you have to know, that there are going to be obstacles on these people's part. They are not going to be able to do what you want them to do. They have to have a fund. It costs money for your people to see you and for your people to get here. Corrie should have included a monthly doctor's visit with the patient, funded by Corrie.

Her big picture, closing thoughts about Corrie:

Take a couple hot shots from each hospital, and bring them here, they are gonna want what y'all did. It's been a good thing for me, positive, rejuvenating. You walk your walk a different way. Life looks different. It feels different. I'm not there yet. I'm not ready to give'em up. Because I feel that I'm back to doing what I'm supposed to do, and I need to finish and this time I'm gonna finish. It did help.

See I don't look at it as an app I look at it as the Corrie program is my heart. It wasn't in any good shape the day she walked in the room. It wasn't. And part of me wanted to give up, and part of me didn't. For other people, not for me. At that point I didn't know how I felt about me, I just knew my heart hurt. That I didn't like it. That I had to do something different. So that quit hurting. And then the Corrie program got introduced. So when I came home, everytime [my partner] said how's your heart, I said the Corrie program's got it, I'm gonna be OK. Because I trusted in the program, because she sold me on it, that it would help me get better. And that was the only way for the first few months that I could think, because otherwise I remembered how it hurt, and I didn't want to remember that. If you asked me that day I would've been crying, I'm trying not to. But it really did, I thought I was going to die, I didn't know if I was makin' it. I just didn't know what was gonna happen. And I think about it all the

\section{Patient's perspective Continued}

time. When I put my watch on in the morning and when I go to work, it's gonna be a good day for Corrie. And when I say that, I'm talking about my heart, because as good as I did, I owe it to the program.

While hospitalised, she was enrolled in Corrie Myocardial infarction, Combined-device, Recovery Enhancement (MiCORE) study, ${ }^{4}$ which was a trial primarily aimed to determine if type I MI patients using Corrie have lower all-cause unplanned 30-day hospital readmissions and related healthcare costs compared with a historical comparison group. Corrie consists of (1) a smartphone application for medication management, education, vitals and care coordination, (2) cooperative sensors including an Apple Watch and an iHealth wireless blood pressure cuff and (3) a data backend platform. This intervention engages patients early during the hospitalisation and facilitates adoption of guideline-directed medical treatment and lifestyle modifications known to improve health outcomes. At the time of enrolment, the patient owned a flip phone and had never used a smartphone. She was provided with an iPhone preloaded with the Corrie app and Apple Watch and wireless blood pressure monitor to participate in the study. She was briefly trained for 30 min on how to use the app, and was provided with an orientation packet highlighting key features of the app.

\section{OUTCOME AND FOLLOW-UP}

With the help of reminders from the Corrie app on both her phone and watch, she tracked adherence to her cardiac medications and follow-up appointments with her primary care doctor and cardiologist (figure 1A,D). She monitored her step count and increased her exercise to climbing stairs and/or walking 3-5 miles daily (figure 1B). She learnt more about cardiovascular health and her recent diagnosis through a curriculum consisting of brief, easy to understand and visually engaging educational videos (figure 1C). She also changed her lifestyle, as recommended in the videos, by quitting smoking, avoiding fast and fried foods, eating heart healthy foods and reducing her soda intake by half. At 30 days, and at an interview conducted 2.4 years after initial enrolment, she was continuing healthy daily habits, avoiding both post-MI complications and hospital readmission.

\section{Learning points}

- A revamp of the hospital discharge process with patientcentred mobile health (mHealth) technology has potential to enhance the myocardial infarction recovery process, in part through improved adherence to guideline-directed medical treatment and lifestyle modifications.

- mHealth is a promising approach to reinforce guidelinedirected medical therapy and lifestyle modification.

- Underserved patients face significant barriers to achieving goals, in part driven by systemic hurdles and in part driven by the high cost of a healthy lifestyle (diet, exercise).

- mHealth typically is unable to reach underserved patients such as ours, due to the cost of digital technology; however, this case demonstrates that mHealth interventions are a promising modality to narrow health disparities, and empower patients in self-management. 


\section{DISCUSSION}

The world of mHealth interventions is at the cusp of a revolution, owing to the rapid adoption of smartphones, smartwatches and other digitally connected health peripherals. In the past few years, researchers have begun to investigate the potential for digital health to enhance the prevention, diagnosis, management and treatment of diseases in nearly all specialties. ${ }^{5-8} \mathrm{mHealth}$ interventions aimed specifically at improving the post-MI recovery process have targeted various aspects of the recovery process, from increasing medication adherence and lifestyle changes, to enhanced vital sign monitoring and digitalisation of cardiac rehabilitation. ${ }^{9-17}$ The electronic modality of such digital health interventions has been varied, and ranges from simple SMS (short messaging service) text messaging platforms, to integrated smartphone app-based and/or web-based portals. The Corrie platform, which our patient used, was a broad integration of these aforementioned features aimed at enhancing patient education, medication adherence, lifestyle changes, vital sign monitoring and care coordination. The Corrie platform's comprehensive approach to recovery, paired with multidevice integration (smartphone, smartwatch, blood pressure cuff), is a unique and powerful approach to augmenting patient engagement and recovery by providing several layers of direct support to the patient. The intangible aspects of patient support (emotional, psychological) are multifaceted, as expressed through the patient's own words in her commentary.

Randomised controlled trials studying mHealth interventions, though identifying benefit in post-MI patients, typically exclude patients who do not already own smartphones, ${ }^{9} 14$ and by extension present socioeconomic bias as wealthier patients are more likely to own smartphones. According to the Pew Research Center, as of 2019 , more than $81 \%$ of adults in the USA owned a smartphone and considering individuals of low socioeconomic status, as defined by a salary of less than US $\$ 30,000$, $71 \%$ owned a smartphone. Meanwhile, only $45 \%$ of individuals in developing nations owned one, leaving $19 \%$ of adults in the USA and 55\% in developing nations without ready access to smartphone-based mHealth interventions. A key implementation issue for mHealth is access to mobile technology from a population health perspective.

There is often a large gap between what the patient does after hospital discharge, and what the clinician counsels the patient to do after hospital discharge. One of the greatest contributors to this disconnect is the inadequacy of the decades-old process of hospital discharge, wherein clinicians verbally communicate postdischarge teaching and instructions within a short period of time, then compile an after visit summary for the patient. mHealth, as showcased in this case and other larger scale studies, ${ }^{4}$ has demonstrated its utility in ensuring proper patient adherence to posthospital discharge instructions, even at 2.4 years follow-up. mHealth interventions are still an unusual novelty, but with active efforts at integration into the healthcare system, will likely become a mainstream channel to modernise antiquated healthcare practices. mHealth has great potential to accelerate our push into 21 st century precision medicine, with tailored medical care that optimises care for each individual patient. One of the greatest advantages of mobile technology is its facile scalability on a population level while remaining personalised to each individual patient's circumstances.

Ultimately, mHealth enabled our patient to transition home safely and improve her health despite a suboptimal hospital discharge system, medical comorbidities, socioeconomic disadvantage and unfamiliarity with smartphones. Her story highlights the potential for mHealth to improve the process of hospital discharge for all patients, including socioeconomically disadvantaged populations new to smartphones. While she obtained a smartphone through the study's loaner programme, patients who cannot afford to purchase a smartphone could theoretically receive assistance through insurance reimbursement or health system investment based on expected savings in healthcare expenditures.

In conclusion, mHealth demonstrates the potential to transform the care of patients after hospital discharge and promote health equity, an approach deserving study and investment.

Acknowledgements We thank Corrie engineering and Lead iOS Developer Ryan Demo for his key technical assistance with figure 1. Matthias Lee, PhD, Erin Spaulding, PhD, and our patient who volunteered her valuable time to contribute to this manuscript to help other patients and guide healthcare system improvement.

Contributors GH, WEY, FAM and SSM each contributed significantly to the planning, conduct and reporting of the work described in the article.

Funding This study has received material support from Apple and iHealth and funding from the Maryland Innovation Initiative, Wallace H Coulter Translational Research Partnership, Louis B Thalheimer Fund, and the Johns Hopkins Individualized Health Initiative.

Competing interests FAM and SSM are founders of and hold equity in Corrie Health, which intends to further develop the digital platform. This arrangement has been reviewed and approved by the Johns Hopkins University in accordance with its conflict of interest policies.

\section{Patient consent for publication Obtained.}

Provenance and peer review Not commissioned; externally peer reviewed.

Open access This is an open access article distributed in accordance with the Creative Commons Attribution Non Commercial (CC BY-NC 4.0) license, which permits others to distribute, remix, adapt, build upon this work non-commercially, and license their derivative works on different terms, provided the original work is properly cited and the use is non-commercial. See: http://creativecommons.org/ licenses/by-nc/4.0/.

\section{REFERENCES}

1 Horwitz LI, Moriarty JP, Chen C, et al. Quality of discharge practices and patient understanding at an academic medical center. JAMA Intern Med 2013;173:1715-22.

2 Ziaeian B, Araujo KLB, Van Ness PH, et al. Medication reconciliation accuracy and patient understanding of intended medication changes on hospital discharge. J Gen Intern Med 2012;27:1513-20.

3 Carroll JK, Moorhead A, Bond R, et al. Who uses mobile phone health Apps and does use matter? a secondary data analytics approach. J Med Internet Res 2017;19:e125.

4 Spaulding EM, Marvel FA, Lee MA, et al. Corrie health digital platform for selfmanagement in secondary prevention after acute myocardial infarction. Circ Cardiovasc Qual Outcomes 2019;12:e005509.

5 Yang Q, Van Stee SK. The comparative effectiveness of mobile phone interventions in improving health outcomes: meta-analytic review. JMIR Mhealth Uhealth 2019:7:e11244.

6 Fedele DA, Cushing CC, Fritz A, et al. Mobile health interventions for improving health outcomes in youth. JAMA Pediatr 2017;171:461-9.

7 Lancioni GE, Singh NN, O'Reilly MF, et al. Smartphone-Based interventions to foster simple activity and personal satisfaction in people with advanced Alzheimer's disease. Am J Alzheimers Dis Other Demen 2019:34:478-85.

8 Werhahn SM, Dathe $H$, Rottmann T, et al. Designing meaningful outcome parameters using mobile technology: a new mobile application for telemonitoring of patients with heart failure. ESC Heart Failure 2019;6:516-25

9 Varnfield M, Karunanithi M, Lee C-K, et al. Smartphone-Based home care model improved use of cardiac rehabilitation in postmyocardial infarction patients: results from a randomised controlled trial. Heart 2014;100:1770-9.

10 Widmer RJ, Allison TG, Lennon R, et al. Digital health intervention during cardiac rehabilitation: a randomized controlled trial. Am Heart J 2017; 188:65-72.

11 Akhu-Zaheya LM, Shiyab Wa'ed Y., Shiyab WY. The effect of short message system (SMS) reminder on adherence to a healthy diet, medication, and cessation of smoking among adult patients with cardiovascular diseases. Int J Med Inform 2017;98:65-75.

12 Cheng K, Oswal A. Smartphone apps and secondary prevention after myocardial infarction - how can long-term usage be improved? Am Heart J 2017;184:e1.

13 Ross ES, Sakakibara BM, Mackay MH, et al. The use of text messaging to improve the Hospital-to-Community transition in acute coronary syndrome patients (Txt2Prevent): intervention development and pilot randomized controlled trial protocol. JMIR Res Protoc 2017;6:e91. 
14 Johnston N, Bodegard J, Jerström S, et al. Effects of interactive patient smartphone support APP on drug adherence and lifestyle changes in myocardial infarction patients: a randomized study. Am Heart J 2016;178:85-94.

15 Treskes RW, van Winden LA, van Keulen N, et al. Using smart technology to improve outcomes in myocardial infarction patients: rationale and design of a protocol for a randomized controlled trial, the box. JMIR Res Protoc 2017;6:e186.
16 Verburg A, Selder JL, Schalij MJ, et al. eHealth to improve patient outcome in rehabilitating myocardial infarction patients. Expert Rev Cardiovasc Ther 2019;17:185-92.

17 Gonzalez M, Sjölin I, Bäck M, et al. Effect of a lifestyle-focused electronic patient support application for improving risk factor management, self-rated health, and prognosis in post-myocardial infarction patients: study protocol for a multi-center randomized controlled trial. Trials 2019;20:76.

Copyright 2020 BMJ Publishing Group. All rights reserved. For permission to reuse any of this content visit

https://www.bmj.com/company/products-services/rights-and-licensing/permissions/

BMJ Case Report Fellows may re-use this article for personal use and teaching without any further permission.

Become a Fellow of BMJ Case Reports today and you can:

- Submit as many cases as you like

- Enjoy fast sympathetic peer review and rapid publication of accepted articles

- Access all the published articles

- Re-use any of the published material for personal use and teaching without further permission

\section{Customer Service}

If you have any further queries about your subscription, please contact our customer services team on +44 (0) 2071111105 or via email at support@bmj.com.

Visit casereports.bmj.com for more articles like this and to become a Fellow 